\section{La subjetividad de la sociedad de trabajo}

\author{
Franz Hinkelammert*
}

\section{RESUMEN}

El ensayo tiene como propósito lanzar una llamada de atención crítica ante una serie de fenómenos de índole política, económica y social que se gestan actualmente en el orden mundial. Describe, de manera amplia, cómo la globalización alimentada por un nuevo capitalismo evolucionado y la burocracia de las trasnacionales socavan la sensibilidad social del trabajo, el núcleo de identidad cultural de los pueblos y la democracia, transformándolas en fraudulentas y marginales, a la vez que contempla los efectos del surgimiento de la dictadura de la seguridad nacional, la guerra antiterrorista y la ley absoluta, que se legitiman a través del miedo y teorías de conspiración fascistas, como medios para imponer un modelo de desarrollo y una ideología, tras los trágicos acontecimientos del 11 de Setiembre.

\section{DESCRIPTORES:}

Globalización. Dictadura de seguridad nacional. Guerra antiterrorista. Ley absoluta. Teologías fundamentalistas.

* Doctor en Economía por la Universidad Libre de Berlín. Tiene estudios posdoctorales en Teología. Entre sus numerosas publicaciones están: Ideologías del desarrollo y dialéctica de la historia (1970), Las armas ideológicas de la muerte (1977), Crítica de la razón utópica (1984), El grito del sujeto (1998), El sujeto y la ley (2003). Rec.24-6-2005. Aprob. 20-2-2006

\section{ABSTRACT}

The purpose of this essay is to criticize a series of political, economic and social issues that are currently present in the world. It widely describes how globalization, fed by a new evolved capitalism and the bureaucracy of transnational companies, undermines the working social sensibility, the cultural identity nucleus of the people and democracy, transforming them in fraudulent and marginal. At the same time, it contemplates the effects of the emergent dictatorship of national security, the antiterrorist war and the absolute law that are legitimated through fear and Fascist conspiracy theories, as means to impose a development model and an ideology, after the tragic events of September 11th.

\section{KEY WORDS:}

Globalization. Dictatorship of national security. Antiterrorist war. Absolute law. Fundamentalist theologies.

\section{INTRODUCCIÓN}

Quiero entrar en nuestro tema por un análisis de la sociedad en la que vivimos y que es una sociedad impregnada por una estrategia de globalización que aplasta la subjetividad del ser humano y que no permite la constitución de la sociedad como una sociedad de trabajo. Más claro vemos este hecho hoy en la tendencia aparecida desde EE.UU., que apunta a la concepción del estado de derecho, en la cual es legalizada la existencia de campos de concentración (como en Guantánamo y otros campos de prisioneros en Irak), la tortura siste- 
mática y la desaparición de personas. Se trata de un fenómeno masivo y el gobierno de EE.UU. lucha por integrar estas medidas en el propio estado de derecho. A lo que aspira el gobierno es a una especie de dictadura mundial de seguridad nacional de EE.UU., integrada en el propio estado de derecho.

Tenemos que ver esta tendencia en el marco de la estrategia de globalización, cómo llega a formularse e imponerse a partir del golpe de Estado en Chile de 1973, del gobierno de Thatcher y del gobierno de Reagan, formulada en el "consenso de Washington" a inicios de los años 80.

Esta estrategia efectivamente global, lo es en nombre de mercados globales en manos de grandes burocracias privadas de empresas transnacionales. La estrategia totaliza los mercados globalmente dadas las nuevas tecnologías (computación, información, transporte) que permiten eso. Se imponen en nombre de la eliminación de las tal llamadas distorsiones del mercado, que son distorsiones desde el punto de vista de estas burocracias privadas, que producen y distribuyen sus productos (y servicios) globalmente. Los “ajustes estructurales son pautas para esta eliminación de distorsiones. Se impone a los Estados y a los gobiernos, convirtiendo la burocracia pública casi en un apéndice de las gigantescas burocracias privadas.

Distorsiones del mercado desde este punto de vista son todas las intervenciones en el mercado con el destino de asegurar universalmente o regionalmente las necesidades humanas. Por eso son distorsiones: las leyes laborales, protecciones legales del trabajo (horas del trabajo, trabajo de niños, protección de la mujer), cualquier política de asegurar universalmente sistemas de salud, de educación, de vivienda, de seguro de vejez, que tienen que ser públicas para lograr universalidad. Pero distorsión es también política de pleno empleo, política de desarrollo en sentido integral, inclusive las políticas de protección del medio ambiente o de autonomías culturales. Pero también es distorsión cualquier control de los movimientos de capitales o de mercancías. En cambio, no es distorsión el control estricto e inclusive violenta de los movimientos de personas humanas.

Es fácil ver esta política de totalización de mercados mundiales en lucha contra gran parte de los derechos humanos, cuyo reconocimiento habían logrado los movimientos populares de emancipación humana desde el siglo XIX. Se marginan o eliminan ahora. Por supuesto, no se logra todo lo que los estrategas pro- 
yectan. Sería la muerte. Pero todo lo que no se logra en este campo, es considerado una imperfección de la estrategia, en cuya perfección se trabaja y se sueña. Se trata de una racionalidad que al soñar produce monstruos.

Sin embargo, esta estrategia produce efectos, que no se han contemplado al imponerla o que no se quieren contemplar. Se hacen visibles en amenazas globales, que ya habían aparecido antes, pero que se profundizan insospechadamente: la creciente exclusión y marginación de grandes partes de la población humana, la subversión de las relaciones humanas mismas y la crisis del medio ambiente son las peores. Se van profundizando precisamente por el hecho de que nuestras burocracias privadas -que además son propietarios de los medios de comunicación- denuncian las medidas necesarias para tomar como distorsiones del mercado. Nos paralizan frente a estas amenazas e impiden una acción eficaz frente a ellas. Estas amenazas no son de por sí apocalípticas. Hay medidas posibles para enfrentarlas. Pero se transforman en apocalípticas si se sigue denunciar estas medidas necesarias como distorsiones del mercado. Eso les da libre paso. Por eso no sorprende que el bloque de apoyo más sólido a esta política en EE.UU. son los apocalípticos del fundamentalismo cristiano, en cuyas filas se incluyen el mismo Bush II y muchos de su junta de gobierno.

Evidentemente, el sistema no tiene ninguna intención de adaptarse o de realizar los cambios necesarios. Pide flexibilidad a todos, pero declara que no va a mostrar alguna flexibilidad. Tiene el lema TINA: There is no alternative (no hay alternativa). Es lo mismo que decir: no vamos a mostrar ni el mínimo de flexibilidad. El capitalismo de la estrategia de globalización efectivamente es el menos flexible de todos los capitalismos que ha habido.

Al no considerar los cambios necesarios, la estrategia se convierte en una máquina aplanadora. Tiene que aniquilar las resistencias y hacerse terrorista. Por tanto, el terrorismo de estado resulta inevitable para poder seguir con la estrategia de globalización. De hecho, estaba ya en sus inicios con las dictaduras de seguridad nacional que surgen en los años 60 y 70 . Se simbolizó por el golpe militar chileno. Hay dos 11 de septiembre que son el marco del proceso. Desde el 11-S 1973 de Santiago, que inició la asunción de la Dictadura de Seguridad Nacional (SN) y a partir del cual se impuso la estrategia de globalización en América Latina 
(AL). Derrocadas las resistencias y los movimientos populares, vino la democratización que va paralela a la imposición de las burocracias privadas y sometidas a sus condiciones. Sin embargo, volvió la resistencia aplastada anteriormente. El segundo 11 de septiembre de Nueva York dio la ocasión para enfrentarla de manera tan irracional como habían sido estos atentados. Ahora "lo único que hace falta" es la guerra antiterrorista igualmente irracional y sin destino.

De esta manera, aparece la tendencia perceptible hacia la recreación de la dictadura de Seguridad Nacional, pero esta vez a nivel mundial y en el interior del estado de derecho. Pero a este nivel hoy resulta imposible hacerlo, sin sostener la vigencia del estado de derecho y de la democracia. La Seguridad Nacional tiene que ser integrada. Eso lleva a la transformación del estado de derecho que está en curso.

\section{La integración de la dictadura de seguridad nacional en el estado de derecho}

Hoy, y especialmente después del 11 de Setiembre de Nueva York, se defiende la inflexibilidad de la estrategia de globalización por la "guerra antiterrorista" y la lucha -mítica- en contra de una conspiración mundial terrorista. Es una lucha que Bush II hasta interpreta como un exorcismo, porque en todas partes se ve enfrentado con la cara del diablo (the evil's face). La inquisición no puede estar muy lejos.

Se trata ahora de una nueva transformación del estado de derecho, que se encuentra en continuidad con las dictaduras de seguridad nacional, que siempre han tenido mucho apoyo de parte de muchos países del norte. Esta dictadura tiene su centro en la tortura, en los campos de concentración establecidos con fines de tortura masiva en función de informaciones (en Chile p.e. Tejas Verdes) y la desaparición de personas.

Si nos basamos en la teoría de Hannah Arendt, se trata de Estados totalitarios.

Ahora aparece la tendencia de introducir este tipo de dictadura de seguridad nacional en el estado de derecho. Efectivamente estamos en camino a una dictadura mundial de seguridad nacional de EE.UU. La tendencia es general y usa el 11$S$ de Nueva York como su pretexto. Se introducen la tortura, los campos de tortura para la información, y la desaparición de personas. Ya hay miles de desaparecidos. Hay una continuidad con las dictaduras de seguridad nacional hasta 
la actual integración de los rasgos más destacados de esta dictaduras en el estado de derecho actual. De Tejas Verdes en el Chile de Pinochet hasta Camp Delta de Guantánamo hay un camino directo.

La misma tortura es legalizada y burocratizada. El Washington Post dice:

Oficiales del Pentágono dijeron que el grupo (un memorando del Departamento de Justicia) examinó por lo menos 35 técnicas de interrogación, y Rumsfield posteriormente aprobó el uso de 24 de ellos en una directiva clasificada del 16 de abril de 2003, que valía para todas las actividades de Guantánamo. El Pentágono rechazó publicar estos 24 procedimientos de interrogación... ${ }^{1}$

Se aprueba y se aplica métodos de tortura, pero el estado de derecho no se mueve. La reacción frente a

1. Pentagon officials said the group examined at least 35 interrogation techniques, and Rumsfeld later approved using 24 of them in a classified directive on April 16, 2003, that governed all activities at Guantanamo Bay. The Pentagon has refused to make public the 24 interrogation procedures... según: Memo Offered Justification for Use of Torture Justice Dept. Gave Advice in 2002 By Dana Priest and R. Jeffrey Smith, Washington Post Staff Writers, Tuesday, June 8, 2004; Page A01 washingtonpost.com las torturas de Bagdad, parece, ha sido más bien por la obscenidad de ellas.

El estado de derecho desemboca en una paradoja. Como estado de derecho declara, que hay territorios y seres humanos, para los cuales no se aplica. Es el homo sacer que es recreado.

Por supuesto, lo nuevo no es que estos estados aplican torturas o las fomentan. A pesar de toda legalidad siempre lo han hecho. En los primeros meses después de la guerra de Irak, la tortura se realizó más bien por un "outsourcing" que entregó esta función más bien a aliados en la guerra. Pero lo mantuvieron en secreto, aunque muchas veces se trataba de un secreto a voces. Pero cada vez más elevan esta medidas a nivel de la legalidad del estado de derecho. Hay sociedades occidentales, que ya lo hacen por medio de declaraciones de la propia Corte Suprema. Pero eso todavía no es lo general. Sin embargo, la tendencia está presente. Hay resistencias de parte de la sociedad liberal. No es seguro el resultado. Pero es llamativo el hecho de que hay muchas fuerzas que impulsan en esta dirección y que incluyen muchos medios de comunicación. Hay conflicto y la actual polarización en EEUU lo atestigua. 
Aparecen teorías jurídicas en el mismo sentido. En el momento, en Alemania se discuten en serio teorías de Günther Jacobs, catedrático de derecho penal de la Universidad de Bonn. Jakobs quiere introducir en el derecho penal la distinción entre el derecho penal para ciudadanos y el derecho penal para enemigos. Las garantías del derecho penal las reserva casi íntegramente para el derecho penal para ciudadanos.

Estas garantías dejan de ser derechos humanos. Para el derecho penal para enemigos en principio desaparecen las garantías. De esta manera se integra la dictadura de seguridad nacional perfectamente en la propia teoría del derecho y, en las definiciones de Jakobs, sería fácil ampliar el ámbito del derecho penal para enemigos a toda resistencia seria a la estrategia de globalización. Jakobs da un camino casi elegante hacia esta meta.

\section{El vaciamiento de la democracia}

Esta transformación del estado de derecho ocurre en sociedades democráticas. Por eso, la integración de la dictadura de seguridad nacional tiene que ser democrática. Reducida la democracia a votación y nada más, necesita una mayoría expresada en elecciones, aunque sean fraudulentas y manipuladas.
Por tanto, se necesita argumentos, que muy bien pueden ser argumentos engañosos y fabricados. Su criterio no es ninguna verdad, sino su capacidad de convencer una mayoría electoral. Aparecen campañas sistemáticas que no cesan nunca. No se menciona siquiera la estrategia de globalización como condicionante de la transformación del estado de derecho.

El medio principal hoy es la declaración de la "guerra antiterrorista". Nos es impuesta por medio de grandes construcciones míticas de sentido, con cosmologías completas y con grandes visiones apocalípticas. Sigue en la huella de las grandes construcciones míticas del fascismo y nazismo del siglo pasado. Aparece de nuevo la construcción de una conspiración mundial, que esta vez es terrorista. Antes era primero judía, con un decisivo acento anticomunista, y posteriormente -después de la II. Guerra Mundial -comunista a secas. Estas construcciones son como materia prima de la construcción de conspiración mundial actual. La actual forma de construcción de la conspiración mundial tiene su antecedente más directo en la construcción correspondiente durante el gobierno de Reagan, que por su parte tiene su antecedente en los mitos del nazismo. 
Reagan creó una cosmovisión del mundo, la cual lo veía polarizado entre EEUU como "la ciudad que brilla en las colinas", es decir, como milenio presente en nuestro mundo, y una conspiración mundial de parte de un reino del mal, que tenía su centro en el Kremlin

de Moscú. Era una réplica visible de la cosmovisión nazi con su milenio en Alemania y la conspiración judía-bolshviki mundial con sede en Moscú, pero también en Manhattan. En el equipo de gobierno de Reagan estaban muchos que ahora vuelven a estar en el equipo de Bush II. Vuelven a luchar en contra de la conspiración mundial ahora formulada entre EEUU como el reino de la libertad y la conspiración terrorista basada en estados canalla, que esta vez tiene la cara del diablo (the evil's face). Esta visión es enriquecidaza por las visiones apocalípticas del fundamentalismo cristiano en EEUU, que hoy es el bloque de apoyo más importante de la derecha republicana. Su ideología encaja sin mayores problemas en este espacio mítico. Por tanto, pueden ser la fuerza mayor que lo sustenta.

De esta manera, la opinión pública es arrollada por la propaganda del miedo. Los argumentos que se basan en problemas reales de la población pierden fuerza y parecen débiles al lado del espanto casi-metafísico del terror que inspiran estas imágenes y mitos de los reinos del mal, del diablo, de fuerzas oscuras que amenazan desde lo desconocido.

Sin embargo, detrás de estos mitos y fantasmas aparece otro argumento, que los sostiene de una manera aparentemente racional. Se trata de un argumento que invierte a lo que los movimientos alternativos sostienen. Estos sostienen una política de afirmación de la vida que exige cambios. Sin embargo, también la imposición ciega de la estrategia de globalización exige cambios. Necesita cambios del poder para lograr superar las resistencias en todas sus formas. Frente a los cambios de la estrategia de globalización que exigen los movimientos alternativos, la ultraderecha en el poder exige cambios en el ejercicio del poder tales que la estrategia puede ser continuada sin mostrar la más mínima flexibilidad. Es un cambio de todas las reglas de convivencia que permita marginar o destruir las resistencias sin respetar alguno de los límites tradicionales del poder. Este cambio ahora esgrime igualmente el argumento de la afirmación de la vida, pero en un sentido contrario a los movimientos alternativos. Se sostiene ahora que este desencadenamiento del poder absoluto del Estado es condición para salvar la 
vida humana, amenazada por el terrorismo y salvada por la imposición de la propia estrategia de globalización y por la integración de parte del estado de derecho de la dictadura de la seguridad nacional. La ultraderecha llama ahora a afirmar la vida amenazada por las resistencias en general, pero especialmente por las reacciones terroristas más bien irracionales.

Ocurre una inversión del argumento de la vida que sostienen los movimientos alternativos, para la cual el terrorismo dio la ocasión: salvar vidas pasa por el dar muerte. La tortura salva vida, los campos de tortura salvan vidas, la desaparición de personas salva vidas, la estrategia de globalización, aunque da muerte, salva muchas más vidas. La propia dictadura de seguridad nacional parece ahora una instancia para salvar vidas.

El argumento es fácil. El terrorismo da muerte. Para salvar las vidas amenazadas, hay que encontrar y eliminar a los terroristas. La muerte de las víctimas inocentes no se puede evitar sino eliminando a los terroristas. Por tanto, cualquier medida para eliminarlos, salva vidas.

Lo mismo en cuanto a la estrategia de globalización. Esta aumentó las tasas de crecimiento del producto, por tanto permite sostener más vidas humanas. Aunque esta estrategia exiga sacrificios humanos, salva como resultado mucho más vidas humanas de lo que sacrifica. $^{2}$

Eso se presenta como realismo de afirmación de la vida. Este realismo promueve la tortura y la desaparición, promueve la explotación y la miseria, pero descubre que a través de ellos afirma la vida de la única manera realista posible.

Desde los inicios de la estrategia, este argumento aparece por todos lados.

En este sentido puede servir una cita de un torturador prominente del Campo Delta en el campo de concentración en Guantánamo, que apareció en el Washington Post:

VanNatta terminó su tarea de superintendente de Camp Delta en septiembre. Dice que hoy está orgulloso de lo que él y sus tropas han logrado.

2. Hayek lo expresa en 1981:

"Una sociedad libre requiere de ciertas morales que en última instancia se reducen a la mantención de vidas: no a la mantención de todas las vidas porque podría ser necesario sacrificar vidas individuales para preservar un número mayor de otras vidas. Por lo tanto las únicas reglas morales son las que llevan al 'cálculo de vidas': la propiedad y el contrato". Hayek, Friedrich von. Entrevista Mercurio Santiago de Chile, 19.4.81 
"Se trata del año más importante que jamás he vivido, porque estoy convencido de que hemos salvado vidas," decía VanNatta, quien ahora volvió para dirigir la prisión de máxima seguridad al norte de Indianapolis.

"Si resulta así como yo creo que resultará, (Camp Delta) será considerado la más única prisión jamás realizada. Si resulta que la información que hemos recolectado salvó vidas, va a ser considerado como uno de lo más adecuado hecho jamás. Sin embargo, si se comprueba de que no ha habido inteligencia (información eficaz), entonces todo será visto como acción de un superpoder que ha usado su poder arbitariamente."3

3. VanNatta ended his tour as superintendent of Camp Delta in September. Today, he says he is proud of what he and his troops accomplished.

"That was the most important year I ever spent, because I think we saved lives," said VanNatta, now back running the maximum-security prison north of Indianapolis.

"If it comes out the way I think it will, it will be viewed as the most unique prison environment ever created. If it comes out that the information we collected did save lives, it will be viewed as one of the smartest moves ever made. If it's proven that there was no intelligence, then it's going to be viewed as a superpower using its power unchecked."
La cita me recuerda una anécdota que se cuenta sobre el Gran Inquisidor Torquemada del siglo XV. Este hizo un diálogo, en el cual se hace a sí mismo la pregunta: Es lícito, no torturar a un hereje? Y contesta: No es lícito no torturarlo porque con eso se le quita su última oportunidad para salvar su alma.

Eso cambia ahora, aunque continua. La pregunta ahora es: ¿Es lícito no torturar a un sospechoso del terrorismo? Y la respuesta: No es lícito no torturarlo, porque con eso se pierde una oportunidad de salvar vidas inocentes.

El memorando citado del Departamento de Justicia dice:

...que las leyes internacionales en contra de la tortura "posiblemente son inconstitucionales (violan la constitución) si son aplicadas a interroga-

\footnotetext{
Torture Policy The Washington Post Company, washingtonpost.com Wednesday, June 16, 2004; Page A26. Staff writers John Mintz, R. Jeffrey Smith and Dana Priest in Washington and David B. Ottaway in Saudi Arabia contributed to this report.

En el drama de Benedetta "Pedro y el Capitán" el Capitán, que es el torturador, habla exactamente el mismo idioma como VanNatta. Ver: Benedetti, Mario: Pedro y el Capitán. Nueva Imagen. Mezico, 1979
} 
ciones" llevadas a cabo en la guerra de Bush en contra del terrorismo..."

Si un empleado del gobierno tortura a un sospechoso preso "lo podría hacer para prevenir ataques futuros de parte de la red al Qaeda en contra de EE.UU.", decía el memorando... escrito en respuesta al pedido de consejo legal de parte de la CIA.4

No torturar resulta ahora ser una violación del derecho y de la constitución, una irresponsabilidad, una colaboración con el terrorismo frente a la cual la tortura es resultado de la afirmación de la vida. Es Torquemada, secularizado.

4. that international laws against torture "may be unconstitutional if applied to interrogations" conducted in President Bush's war on terrorism, according to a newly obtained memo...

If a government employee were to torture a suspect in captivity, "he would be doing so in order to prevent further attacks on the United States by the Al Qaeda terrorist network," said the memo, from the Justice Department's office of legal counsel, written in response to a CIA request for legal guidance. It added that arguments centering on "necessity and self-defense could provide justifications that would eliminate any criminal liability" later ... según: Memo Offered Justification for Use of Torture Justice Dept. Gave Advice in 2002 By Dana Priest and R. Jeffrey Smith, Washington Post Staff Writers, Tuesday, June 8, 2004; Page A01 washingtonpost.com
Este tipo de argumento aparece por todos lados. Apareció también en relación con la bomba atómica sobre Hiroshima. En una entrevista el piloto responde la pregunta sobre lo que ha sido lo más importante en su vida:

Obviamente el haber formado y operado el grupo 509, entrenado para usar la bomba... Originariamente se me dijo que los bombardeos en Europa y Japón se iban a hacer simultáneamente.

Me atrevo a decir que salvé millones de vidas al hacerlo, labor que me tomó diez meses y medio. ${ }^{5}$

La tortura, la bomba atómica, la estrategia de globalización: todo salva vida. Hasta habría sido irresponsable e inconstitucional no haber lanzado la bomba atómica sobre Hiroshima.6

5. Entrevista con Paul Tibbets, coronel, quien con 27 años de edad como piloto principal tiró la bomba atómica de Hiroshima el 6 de agosto de 1945. La bomba se llamaba Little Boy y el avión llevaba el nombre de la madre del piloto principal Enola Gay.

La entrevista la hace Andrés Jiménez, periodista de la revista colombiana Semana. Reproducida en La Nación, 22.8.99

6. Se trata de un argumento que es heredero de argumentos conservadores anteriores. Por ejemplo, decía el Cardinal Höffner, Erzobispo de Colonia en Alemania: 
El terrorismo del estado se hace pasar como un realismo humanista, verdadera afirmación de la vida humana.

Con eso el argumento es completo. Se trata de un círculo argumentativo, cuya estructura lo encontramos igualmente en todos los totalitarismos anteriores del siglo XX. No hay más que variaciones.

Tiene una fuerza muy grande para arrastrar masas a las cuales se ha logrado inculcar el miedo correspondiente. Hace desaparecer la realidad y desata una fuerza agresiva inimaginable y sin límites. Cualquier crítica e incluso llamado a la calma parece una simple traición y una falta completa de realismo.

Si se impone, arrastra la democracia desde adentro y la transforma en una estructura hueca al servicio de poderes incontrolables del asalto al poder mundial.

"El derecho del Estado de aplicar la pena capital (derecho de la espada) es un reconocimiento especial de la invulnerabilidad de bienes humanos, en especial de la vida humana. La Santidad del orden de Dios se muestra 'con poder' en este Eon por medio de la pena capital." Höffner, Josef: Christliche Gesellschaftslehre, Kevelaer 1975 p. 231

\section{La subjetividad de la sociedad y su aplastamiento}

Ha surgido un sistema gigante de una producción inmensa de riquezas, pero que resulta ser a la vez un sistema de destrucción progresiva del ser humano y de la naturaleza externa a este ser humano. Se hace presente esta destructividad en las amenazas globales de la exclusión, de la subversión de la relaciones humanas y de la destrucción del ambiente de la vida humana. Este sistema desarrolla hoy una agresividad creciente para poder dominar los reacciones de resistencia que provoca. La violencia es presentada como solución y el aniquilamiento de los adversarios como única salida para poder sostener la inflexibilidad del sistema. El sistema se hace presente como la ley absoluta en nombre de la cual aplasta. Aniquilamiento y exterminio dominan las escenas de la lucha por el poder. Lo vemos en muchas partes: Afganistán, Irak, Colombia.

El sistema se presenta como ley absoluta, inflexible, sin límites y, por tanto, con derecho a todo. Se presenta como la justicia y la libertad. Dersde la Edad Media vienen dos proposiciones importantes sobre lo que significan tales leyes absolutas. Las quiero analizar brevemente: 
1. Summa lex, maxima iniustitia. (Ley absoluta, máxima injusticia). La ley tomada como ley absoluta no es justicia, sino injusticia. Al llamarse justicia, traiciona la justicia.

A eso se junta una segunda proposición:

2. Fiat iusitia, pereat mundus (que se aplique la justicia aunque perezca el mundo). Al buscar la justicia en la ley absoluta, se hace hasta perecer el mundo.

Se trata de dos proposiciones, que ya tenían validez en la Edad Media, pero que la tienen hoy en un grado infinitamente mayor.

Estamos en eso. El mundo está por explotar en nombre de una ley absoluta, cuyo cumplimiento amenaza hasta la existencia de la tierra.

Aparecen resistencias, que son de muy variado tipo. Van desde reacciones irracionales y violentas - tan irracionales como el mismo sistema - hasta las resistencias populares que se están extendiendo por el mundo entero.

En estas resistencias populares aparece el reclamo de la recuperación del ser humano como sujeto -sujeto corporal y necesitado-, que tiene que ser la última instancia de cualquier sistema social. Que no lo es, pero tendría que serlo, para que el mundo siga siendo un mundo vivible.
La discusión es amplia y lo es desde décadas. Participan también las iglesias. Sin embargo, quiero presentar algunos aportes que me parecen valiosos y que vienen desde la doctrina social de la Iglesia católica.

Creo que la subjetividad, de la cual se trata, está muy presente ya en lo que dice el Papa Pablo VI:

Si la tierra está hecha para procurar a cada uno los medios de subsistencia y los instrumentos de su progreso, todo hombre tiene el derecho de encontrar en ella lo que necesita (n. 22)...

Todos los demás derechos, sean los que sean, comprendidos en ellos los de propiedad y comercio libre, a ello están subordinados: no deben estorbar, antes al contrario, facilitar su realización, y es un deber social grave y urgente hacerlos volver a su finalidad primera (n. 22). ${ }^{7}$

El concepto de la subjetividad de la sociedad adquiere ahora precisamente este sentido: ninguna ley puede ser absoluta y toda ley tiene su última instancia en la vida de cada una de las personas huma-

7. Pablo VI: Encíclica Populorum progressio.

La solidaridad universal, que es un hecho y un beneficio para todos, es también un deber (n. 17). 
nas. Juan Pablo II usa para esta exigencia el término de la "subjetividad de la sociedad":

En medio de esa múltiple interacción de las relaciones vive la persona y crece la "subjetividad de la sociedad". El individuo hoy día queda sofocado con frecuencia entre los dos polos del Estado y del mercado. En efecto, da la impresión a veces de que existe sólo como productor y consumidor de mercancías, o bien como objeto de la administración del Estado, mientras se olvida que la convivencia de los hombres no tiene como fin ni el mercado ni el Estado, ya que posee en sí misma un valor singular a cuyo servicio deben estar el Estado y el mercado (n. 49). ${ }^{8}$

Y lo vincula con la sociedad del trabajo con el término "primacía del trabajo al capital"9. En este sentido aparece la tesis de la subjetividad de la sociedad, que se basa en la primacía del trabajo al capital.

Creo, que se trata de aportes que siguen vigente. Sin embargo, son a la vez aportes que son abandonados en muchas teologías actuales. Quiero mencionar tres:

8. Juan Pablo II: Encíclica Centesimus annus.

9. Juan Pablo II: Encíclica Laborem Exercens
1. la teología de la prosperidad. Es la teología de un sujeto que se deja aplastar por el sistema y busca en la riqueza de por sí su afirmación de la gracia de Dios.

2. las teologías apocalíticas del fundamentalismo cristiano. Son teologías de aceptación de la catástrofe que el propio sistema está produciendo. Son teologías de la aceptación de esta catástrofe como tribulación, que antecede a la segunda venida de Jesús. No ven remedio para esta catástrofe y denuncian cualquier esfuerzo de enfrentarla como acción del anticristo. Es teología de un sujeto aplastado por el sistema, que se identifica con él sin límites, interpretando la destructividad del sistema como camino de Dios.

3. las teologías y éticas de los valores morales individualizados. Vuelve una moral, que se desentiende de todos los grandes problemas morales. En las recientes elecciones de EE.UU. el $22 \%$ de los electores dijeron, que habían votado por la recuperación de los valores morales. Entre estos valores no había ninguna preocupación por los grandes genocidios que están en curso en Irak y que, a raíz de esta votación por valores morales, se van a extender a otros países. Tampoco había preocupación por la creciente 
pobreza en la población humana ni por la destrucción de la naturaleza. Estos problemas parece que no afectan a los valores morales por recuperar. Se trata de valores más bien vinculados con la sexualidad humana. Desaparecen los grandes crímenes de nuestro tiempo y son desaparecidos en nombre de estos valores morales individualizados. El problema de los matrimonios de homosexuales parece mucho mayor que cualquier genicidio cometido o por cometer. ${ }^{10}$

Son teologías fundamentalistas, pero su vigencia va muchas veces mucho más allá de los grupos explícitamente fundamentalistas. Es notable que hoy las enseñanzas de la doctrina social, que mencioné, sobreviven más bien en grupos cristianos de base y entre teólogos de liberación. Las cúpulas de la propia iglesia católica las han abandonado, aunque sin suprimirlas. Pero no tienen mayor vigencia hoy en las declaraciones de esta iglesia. También se concentra en valores morales individualizados. Centesimus annus fue la última

10. “En efecto, la cólera de Dios se revela desde el cielo contra toda impiedad e injusticia de los hombres que aprisionan la verdad en la injusticia; pues lo que de Dios se puede conocer, está en ellos manifiesto: Dios se lo manifestó." Rom 1.18 (Jerusalén) gran encíclica social, que recordaba la primera, que era Rerum Novarum. Terminaron 100 años de doctrina social, pero después de estos 100 años esta doctrina parece más bien enterrada.

La tarea me parece más bien, seguir esta tradición o recuperarla, elaborarla para nuestro tiempo y participar de esta manera en el gran movimiento de resistencia frente al sistema creado por la estrategia de globalización. Hay elementos que aportar, pero estos quedan neutralizados si no se los aporta efectivamente.

\section{BIBLIOGRAFÍA}

Hinkelammert, Franz, 2003, El sujeto y la ley, Heredia, Ed. EUNA.

Hinkelammert, 1998, El grito del sujeto, San José, Ed, DEI,

Iribarren, Jesús, y otros (Editores), 1976, Ocho grandes mensajes, Madrid: Biblioteca de autores cristianos.

Juan Pablo II, 1991, Centesimus annus, Roma, Ed. Ciudad del Vaticano

Juan Pablo II, 1987, Sollicitudo rei socialis, Roma, Ed. Ciudad del Vaticano.

Juan Pablo II, 1981, Laborem exercens, Roma, Ed. Ciudad del Vaticano. 